\title{
Research on social representation of network hot words in digital era
}

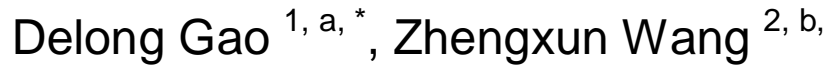

${ }^{1}$ Center for Digitalized Culture and Media, University of Electronic Science and Technology of China, Chengdu, Sichuan, China

${ }^{2}$ School of Political Science and Public Administration,University of Electronic Science and Technology of China, Chengdu, Sichuan, China

Asin205@foxmail.com, bdavid.printf@qq.com

* Correspondence author

Keywords: Network hot words, Social representation, Popularity, Social mentality

\begin{abstract}
The deformation of language is always accompanied by the transformation of times; the form and combination of "Chinese characters" are always characterized with the features of the times since its birth, it also has the characteristics of social order and social formation and evolution embodiment. In the current Internet era, the Internet brings about gigantic transforms in human's life and communication. The network popular language molds the new language expression and the present situation of writing style. A new sort of vocabulary information utilization and interactive mode enables the online and offline communication more rich, vivacious and even imaginative. The stylistic change of network language and the social mentality behind the expression modes are investigated from the overall concept and characteristics of the network hot words along with the epidemic reasons.
\end{abstract}

\section{Introduction}

The invention of characters overwhelmingly expands the possibility of inheriting and continuing the human civilization. It defines the historical records and systematizes the people's life. The "Analytical Dictionary of Characters" of Xu Shen in Late Eastern Han Dynasty can be said as a positivism research with modern significance from a certain angle. Language is the first significant leap in the process of deepening human communication and dissemination. However, the "network hot words" with Internet as platform show a blowout eruption trend at the moment, its meaning goes far beyond the literal meaning of the word itself and becomes a synonym for social phenomena of some attitudes and opinions of netizens[1]. By techniques of irony, parody and harmonic tone, hot words form new words which are different from original intention in manner of network interaction channels, it emphasizes or implies the meanings of questioning and criticizing, etc. with strong property of entertaining the masses and black humo[2]r. The definition, types and causes of formation of network hot words are analyzed in this paper based on the theory of social representation so as to discuss the mapping of social mentality in the language deformation of ontology of Internet catchwords. The social representation theory that put forward by the French psychologist Moscow Veitch is used to express the group's perception or consensus about something with sharing acts, which embodies a social mentality[3]. Only in the concrete combination of social, historical and cultural background with events and cases can something be better understood.

\section{Social expression features of network hot words}

There are many definitions of network hot word, integrating definition of various concepts, it is exactly the language which created by netizens and arisen from the Internet medium with social hot spot phenomenon and hot events as well as the "eye-catching" phenomenon of daily life as objectives to disseminate through online and offline interaction. It often involves all aspects of human life, such as politics, economy, culture, environment and social life from the perspective of 
social structure and function. Network users create the network hot words firstly in order to summarize the hot social phenomena or events and then cause the reasons of concern so as to form the interaction of public opinion to affect the reality. That is to say, a symbol is needed to refer to this event or phenomenon for the Internet users to conduct convenient description and communication when there are certain social phenomena or news. The netizens capture the core of an event or phenomenon and sum up a concise word with high generalization to utilize it in the network by analyzing. The network hot words come into being when this word is widely used in actual network communication and extensively spread in social life, which may reflect the views and tendencies of most netizens to some extent. It is also argued that the Internet hot word is a typical expression of public opinion, and its expression purposes and modes have distinctive characteristics of subculture communication[4].

\subsection{The universality of network dissemination}

The network hot words, which are different from the canonical general language system, have been erupted and popular with exponential function trend in recent years. Taking an overall view of these network hot words, they have universality, including the universality of the source and dissemination; and there is an increasingly clear universality of utilization on this basis. The sources of network hot words have universality, involving political, economic, cultural, entertainment, and military and other fields. Its universality of the source cannot leave the numerous social information which is represented by network hot words. With the development of communication means in "global village" as well as the openness and globalization of information exchange between people and people, man and things, regions and regions, state and state, the network hot words have the universality in dissemination, in which the case is that the network hot words of one country may also be spread and popular in another country. Network hot words are not the "silent" dissemination of simple text language; people's interpersonal oral communication in the real world increasingly highlights the universality of use of network hot words, it embodies the sense of intimacy of utilization, narrows the distance between speakers and generates the common meaning space of network hot words. More and more people in current Internet era may have access to the network hot words and extend them from the network environment to the real society so as to have greater impacts on society.

\subsection{Novelty of utilizing perception}

There is a profound and creative language disinterment in the process of the generation of network hot words, thus they are naturally novel. The novelty of network hot words embodies two kinds of "use and satisfaction" mentalities in the using process of netizens; firstly, it is some curiosity or prying psychology about the Internet hot word, its novelty often causes netizens' curiosity, attention and citation, communication. Secondly, it is the prominent expression of self-emotion in the process of using; the novel network hot words may express the "resonance" mood of netizens at that time or express their entertainment mentality, etc. Therefore, the network hot words with novelty are associated with a certain time, which can reflect the characteristics of the times with new and fashionable features. Network hot words are usually generated by creation or adaption by netizens, and thus this sort of traditional language structure is often broken; it is more casual, novel and humorous in forms.

\subsection{The timeliness of speech deformation}

The ontology of network hot words is still language; hot words may mean that they may be "frozen". Network hot words are characterized with timeliness in respect of canonical language system. A large number of network languages may be in vogue or even be eliminated without a trace in a short period. Of course, language is developing; thus there will be a certain amount of network hot words re-incorporated into the canonical language system, for example, the word "gelivable" causes the language discussion with the headline in "Peoples Daily", and it is then incorporated into the canonical language use system. Secondly, the Internet usage group is mainly the young people who are characterized with open mindedness, the pursuit of fashion and more 
innovative ability. Network hot words are mostly popular for short of time, the new hot words may continue to shock and replace the old ones, maverick young people are more likely to keep up with fashion trends to use the current hot words; and the old words may slowly fade out of sight and disappear from the scene in a few months as the number of usage decreases.

\subsection{Conciseness of thinking cognition}

Simplicity and clearness are another features of the network hot words; the concision and perspicuity on the basis of generality are the necessary magic weapons for the survival of network hot words. Because of the particularity of network and the need for network communication speed, the network hot words are more concise and understandable than the traditional language; it is more inclined to use distinct text to facilitate the audience to receive and understand in the process of communication. Of course, the process of the propagation of network hot words is also accompanied with the dissemination of the paraphrase of network hot words, which is of vital significance to the conciseness of network hot words. The process of a network hot word from "generation" to "widespread" is always accompanied with the dissemination process of "parabolic" type. The dissemination of the paraphrase of network hot words may promote the "parabolic power", and it also determines the vertex of the parabola in respect of the conciseness of network hot words; pursuing the terse and demotic style, the hot words are often not decorated with flowery rhetoric, only straightforward and accessible modes instead; the conciseness can also be understood as more colloquial.

\section{Reasons for the prevalence of Internet Language}

\subsection{Group psychology under hot events}

The concept of social hot event still has no exact authoritative explanation, but it is roughly defined as an off line event that draws wide attentions in public opinion, exerts a certain influence on society, spreads over many medias and forms a public topic over a period of time. And the Internet hot words generated by disseminating the hot events on the mobile Internet platform shall be the "tagged" vocabulary generated from the attention and discussion on this offline events in network. "Hot event" in the Internet hot words is another expression of the arguments about events for netizens; it can be the original words of the parties in the event or the word associated and imagined by netizens. Hot event with the group psychology exactly refers to that the acts upon our own perceptions on things can conform to the universal standards and norms that the public can afford. Each individual living in the society exactly has a certain group psychology to a certain extent; everyone wants to keep up with the "popular" pace of society and times. Therefore, the group psychology will prompt him to accept and spread the catchword when it is gradually gaining popularity among the general public, otherwise the social individual may pretend to face the pressure from society. Thus, the group psychology may accelerate the spread of catchword and expand its scope of dissemination.

\subsection{Language games under hot broadcasted videos}

Network hot words in hot broadcasted videos are characterized with more magnanimity, more openness and more interest. It is pointed in the article "Film and television drama and barrage have become the "creation base" for network hot words" of Wen Wei Po that the film and television dramas make a "great contribution" for the current network popular hot words. Hot broadcasted film and television contribute the magnanimity and openness of re-creation for the network hot words. Serious drama, shocking drama and network drama all make contributions; massive videos make it possible for network hot words to possess the magnanimity, its variety and abundance of videos also make the re-creation of netizens with openness. In the popular online drama "Absolutely unexpected", Tang Priest refuses to pilgrimage to the West with a reason of "I just still want to be a handsome man quietly"; and the "handsome man" is endowed with the meaning of "euphemistic refusal" and "self-mockery and narcissism". Game is a mode to relieve stress in daily leisure time. 
Playing game can be described as an instinct as people may soon learn how to play games. Utilizing the catchwords in games can be drain the stress and obtain happiness.

\subsection{Stress catharsis in social networking site}

The common social networking sites may refer to micro-blog, tieba, forums and other social tools; a large number of network hot words are born hereof. Micro-blog hot topic list can be said to have become the barometer and hot event broadcast device for Internet users; a large number of network hot words generate and are widely used. In the trans-conforming China, people in the society are faced with various pressures. Language venting becomes the best way to relax the stress under the social pressure and environmental constraints around. In addition, people exchange the information more and more frequently, and their brevity and quick-pursuing psychology make them more concise in using language, and this succinct style is very popular with young people. In the "fragmentation" era, social structural pressures push the fast rhythm, emotionality and symbolization to the extreme. "Fast" flows in the superficial-ness of surface information, which is the characterization of unconscious, impetuous, blind and confused mentalities in society.

\section{Network hot words: reflection of social mentality}

Behind the use of network hot words is the reflection of the emotional tendency and expression of the whole netizen group, which is the netizens' social mentality. Netizens' social mentality is the sentiment expressed by their opinions about an event or phenomenon, the mood state of the whole netizen group for a certain period, a sort of refraction of social reality, a window that reflects the relationship between individuals and groups, society and the nation[5].

\subsection{Pan-entertainment spoof and collective anxiety relief}

High housing prices, high commodity prices, difficult employment and all kinds of life pressures pound people's hearts with the continuous development of society and continuously quick circadian rhythm, showing a widespread anxiety. And such an open platform of Internet offers the opportunity to ease the anxieties; people create, spoof and use the Internet hot words effected by entertainment consciousness, they get releases in the Internet world. People are eager to release their inner pressure through entertainment, and their anxiety is relieved and the pressure is released through light chats and humorous utterances as the network hot words are interesting. The impact of a variety of life stress makes people more and more dependent on the Internet to forage happiness and relieve anxiety and depression moods. The popular Internet hot words in 2015, such as "heart is plugged", "really drunk", "I want to be quiet" and so on, are often used by netizens in communication and interaction, thus their emotions and feelings are expressed with humor. The use of these hot words increases the interest of chatting and adds a pleasure to the ordinary boring life to some extent.

\subsection{The omission of mainstream values of the pluralistic thought trend}

The hot words from "Your mother call you home for dinner," "do not obsess me, I am just a legend" to "I just want to be a quiet pretty man", "which excavator is strong? Looking for Lan Xiang in Shandong, China" doesn't seem to make any real sense but are wantonly sought after by netizens, which reflects the their empty, bored and impetuous inner world to some extent. One of the main reasons for this occurrence is the absence of mainstream values. The single value system is broken and presented as a state of pluralistic coexistence under the pluralistic background. This state, on the one hand, brings about the surge of ideas and renewal of ideas to socially, and also causes the conflict between mind and the psychology, which may force people to adjust their value attitudes and choices. In face of various intertwined values, the absence of mainstream values causes people to lose the original frame of reference in value analysis and choice so that the perplexed and alarmed, uncomfortable state of mind generate. 


\subsection{Structural negative phenomena focus and the display of public opinion supervision}

Network hot words reflect the focused hot spots and livelihood issues during a period of time as well as an event or problem of a country or region that receives widespread attention during this period, which has the temporal nature. The concerns about the negative social focus attention may help the Internet to play the supervision role of public opinion; it replaces the previous news headlines, stays at people's minds and subverts the traditional way of obtaining news with the expansion of network coverage and the popularity of network hot words. These hot words are directed at the politics of current affairs, anecdotes, livelihood phenomena; they become an indispensable part of people's daily life by right of the characteristics of conciseness, humor, catchy property, keeping up with the times; it also contributes to the transformation of the supervision of public opinion from the mass media dominating to the mass dominating. Public opinion supervision, especially the network supervision by public opinion, as a social supervision mode with low cost and high efficiency, its guiding function becomes harder to ignore.

\subsection{The attention to political topic and promotion of political participation}

The netizens show a rising trend of concern and participation in politics from some popular network hot words in recent years. Especially since the central authorities have determine to make great efforts on anti-corruption, the hot words such as "hit tiger"," shoot fly" and "hunt fox" become popular, reflecting the ultra high degree of recognition for anti-corruption actions. The survey shows that netizens are most satisfied with the issue of anti-corruption; at present, most netizens take an optimistic and affirmative attitude towards the series of anti-corruption actions of central authorities. They express that "corruption comes from power" and it needs the "effective restraint"; it shall rationally utilize the power under the legal norms and curb the breeding of corruption from the roots. As the anti-corruption efforts are deepened currently, "official-business transactions of closed type", "small officer with huge corruption" and "hilltop-ism" become the new network hot words to criticize the anti-corruption. These "hot words" accurately point to corruptions in some departments, regions and fields, it also exposes the deficiencies of vulnerabilities and systematic-ness. Participation in the democratic process is more deepening with the public attention rate to politics.

\section{Conclusions}

Internet catchword is a linguistic phenomenon, and it is more a cultural phenomenon and social phenomenon. What it reflects is the social life; it is the dissemination and expression of the evolution of social order, the pulse apparatus to grasp the change and transition of society. Network hot word is a sort of thinking rather than a simple communication tool. When expressing our meaning, the network hot words also depict our thinking, deepen our thinking mode, and even solidify or restrict our thinking. Borrowing the "Chibi Fu" of Su Shi, the network hot word can be also described as that it possesses the colors as soon as the eyes see; it becomes the sound as soon as the ears hear; it is shaped as soon as the body touches. Social mentality of current netizens are concluded through the analysis on the utilizing motivation of network hot words, that is, "universal entertainment spoof consciousness", "the lack of mainstream values under the pluralistic ideological trends", the focus on negative social hot spots" and "promotion of attention rate to politics"; the universal entertainment spoof consciousness can effectively relieve the anxiety; the focus on negative social hot spots is conductive to play the supervision role of public opinion and promote the attention rate to politics. It is of realistic significance to comprehend the relationship between language and society that probing into the transition and evolution of social mentality from the network hot words.

\section{References}

[1] Xue Guolin, Liu Zhijie. A curvilinear expression of opinions. the social attitudes reflected by 
"network hot words" [J]. Journalists, 2009, (06): 20-22.

[2] Fang Ting, fan Yingli. Interpretation for the grassroots cultural characteristics from network hot words, [J/OL]. News enthusiast, 2011, (21): 11-12.

[3] Guan Jian, Yue Guo An. Social representation theory and its development [J]. Journal of Nanjing Normal University (Social Sciences Edition), 2007, (01): 92-98. [2017-09-06].

[4] Li Tiechui. Network hot words: a expression of public opinion for dissemination of subculture. Contemporary dissemination.2015

[5] Weng Ping. Study on the characteristics of Internet communication language and its social psychology [J]. Journal of Guangxi University for Nationalities (Philosophy and Social Sciences Edition), 2006, (S1): 246-247+287. [2017-09-06]. 